\title{
A note on the implications of factorial invariance for common factor variable equivalence
}

\section{Michael Maraun \& Moritz Heene}

To cite this article: Michael Maraun \& Moritz Heene (2015): A note on the implications of factorial invariance for common factor variable equivalence, Communications in Statistics Theory and Methods, DOI: 10.1080/03610926.2014.917186

To link to this article: http://dx.doi.org/10.1080/03610926.2014.917186

Accepted author version posted online: 03

Nov 2015.

Submit your article to this journal

Џ Article views: 4

Q View related articles $\widetilde{ }$

View Crossmark data $\asymp$ 


\title{
ACCEPTED MANUSCRIPT
}

\section{FACTORIAL INVARIANCE}

\section{A note on the implications of factorial invariance for common factor variable equivalence}

Michael Maraun ${ }^{1}$, Moritz Heene, ${ }^{2 *}$

${ }^{1}$ Department of Psychology, Simon Fraser University, Burnaby, Canada

${ }^{2}$ Learning Sciences Research Methodologies, Ludwig Maximilian University, Munich, Germany

Correspondence: Prof. Dr. Moritz Heene, Ludwig Maximilian University, Munich, Germany,

Learning Sciences Research Methodologies, Leopoldstr. 13, D-80802 Munich, Germany, heene@psy.lmu.de

Original date of receipt: 22-Jul-2012

\begin{abstract}
There has come to exist within the psychometric literature a generalized belief to the effect that a determination of the level of factorial invariance that holds over a set of $\mathrm{k}$ populations $\Delta \mathrm{j}, \mathrm{j}=$ $1 . . s$, is central to ascertaining whether or not the common factor random variables $\xi \mathrm{j}, \mathrm{j}=1 . . \mathrm{s}$, are equivalent. In the current manuscript, a technical examination of this belief is undertaken. The chief conclusion of the work is that, as long as technical, statistical senses of random variable equivalence are adhered to, the belief is unfounded.

Keywords

Factorial invariance, random variable equivalence, multigroup confirmatory factor analysis, latent variable, manifest variable.
\end{abstract}




\section{ACCEPTED MANUSCRIPT}

\section{Introduction}

Consider the research context in which random $p$-vectors, $\mathbf{X}_{j}, j=1$..s, are distributed over each of $k$ populations $\Delta_{j}$ of objects. Define the $p$ by $p$ covariance matrices $\boldsymbol{\Sigma}_{j}=\mathrm{E}\left(\mathbf{X}_{j}-\boldsymbol{\mu}_{j}\right)\left(\mathbf{X}_{j}-\boldsymbol{\mu}_{j}\right)$ and mean vectors $\boldsymbol{\mu}_{j}=\mathrm{E}\left(\mathbf{X}_{j}\right), j=1 . . s$. Although, of course, there is no necessity that it be so, let it be the case that each $\mathbf{X}_{j}$ happens to be representable by the unidimensional, linear factor modeli; i.e.,

$$
\mathbf{X}_{j}=\boldsymbol{\tau}_{j}+\boldsymbol{\Lambda}_{j} \xi_{j}+\boldsymbol{\delta}_{j}, j=1 \ldots s,(1)
$$

wherein $\xi_{j}$ is a common factor random variable and $\boldsymbol{\delta}_{j}$ is a $p$-vector of random uniquenesses. The parameters of the representation are $\boldsymbol{\Lambda}_{j}$, a $p$-vector of regression coefficients (factor loadings), $\boldsymbol{\tau}_{j}$, a $p$-vector of intercepts, $\boldsymbol{\Theta}_{\delta}$, the $p$ by $p$, diagonal, positive definite, covariance matrix of $\boldsymbol{\delta}_{j}$, and $\kappa_{j}$, the mean of $\xi_{j}$. It is fundamental to linear factor analytic representations that $\mathrm{E}\left(\boldsymbol{\delta}_{j}\right)=\mathbf{0}$ and $\mathrm{C}\left(\xi_{j}, \boldsymbol{\delta}_{j}\right)=\mathbf{0}, j=1 . . s$. A first identification constraint is that $\mathrm{V}\left(\xi_{j}\right)=1, j=1 . . s .{ }^{\mathrm{ii}}$

If each $\mathbf{X}_{j}$ is representable as in (1), then the following factor- and mean structures hold in the populations $\Delta_{j}, j=1 . . s$ :

$$
\begin{aligned}
& \boldsymbol{\Sigma}_{j}=\boldsymbol{\Lambda}_{j} \boldsymbol{\Lambda}_{j}{ }^{\prime}+\boldsymbol{\Theta}_{\boldsymbol{\delta} j} ; \text { (2) } \\
& \boldsymbol{\mu}_{j}=\boldsymbol{\tau}_{j}+\boldsymbol{\Lambda}_{j} \boldsymbol{\kappa}_{j} .
\end{aligned}
$$

With Meredith's (1964) and Joreskog's (1971) invention of Multigroup Confirmatory Factor Analysis (MGCFA) and the extensions made by Sorbom (1974), researchers were provided easily implementable statistical tools that could be employed to test hypotheses pertaining to the cross-population invariance of one or more of the parameters $\boldsymbol{\Lambda}_{j}, \boldsymbol{\tau}_{j}, \boldsymbol{\Theta}_{\delta j}$, and $\kappa_{j}$. Various levels of factorial invariance were defined, perhaps the most oft-tested forming the following nested hierarchy: i) configural invariance. $\boldsymbol{\Lambda}_{j}, j=1 . . s$, have their null elements in the same 


\section{ACCEPTED MANUSCRIPT}

locations (Thurstone, 1947); ii) weak or pattern invariance. $\boldsymbol{\Lambda}_{j}=\boldsymbol{\Lambda}, j=1 . . s$ (Thurstone, 1947); iii) strong factorial invariance. $\boldsymbol{\tau}_{j}=\boldsymbol{\tau}, \boldsymbol{\Lambda}_{j}=\boldsymbol{\Lambda}, j=1 . . s$; iv) strict factorial invariance. $\boldsymbol{\tau}_{j}=\boldsymbol{\tau}, \boldsymbol{\Lambda}_{j}=\boldsymbol{\Lambda}$, $\boldsymbol{\Theta}_{\delta j}=\boldsymbol{\Theta}_{\delta}, j=1 . . s$. For the sake of clarity, it should be noted that strong and strict factorial invariance were subsequently considered by Meredith (1993) using a structural equation approach due to both Joreskog (1971) and Sorbom (1974).

A survey of the literature suggests the existence of two classes of beliefs about why the issue of factorial invariance is empirically important. Class 1 explanations cite an extensive literature (cf. Dolan, Roorda, and Wicherts, 2004; Horn and McArdle, 1992; Meredith and Teresi, 2006; Millsap, 1997; Millsap and Kwok, 2004; Millsap and Yun-Tein, 2004; Vandenberg and Lance, 2000; Wicherts, Dolan, and Hessen, 2004; Wu, Li, and Zumbo, 2007) that seems to establish that cross-population quantitative comparisons of particular of the parameters of the distributions of the $\mathbf{X}_{j}, j=1 . . s$, can be coherently undertaken only given the realization of particular levels of factorial invariance. Thus, for example, it is taken as established that coherent quantitative comparisons of the mean vectors $\boldsymbol{\mu}_{j}, j=1 . . s$, can only be undertaken when a level iv invariance holds (Byrne and Watkins, 2003; Lubke, Dolan, Kelderman, and Mellenbergh, 2003; Meredith, 1993; Meredith and Teresi, 2006). Class 2 explanations, on the other hand, rest on the belief that cross-population invariances in $\left\{\boldsymbol{\Lambda}_{\mathrm{j}}, \boldsymbol{\tau}_{\mathrm{j}}, \boldsymbol{\Theta}_{\mathrm{\delta j}}, \kappa_{\mathrm{j}}\right\}$ bear on the issue of whether the common factor random variables $\xi_{j}, j=1 . . s$, are equivalent, and, in particular, that a level iv invariance establishes the equivalence of the $\xi_{j}, j=1 . . s$.

However, a moment's consideration reveals that Class 1 and Class 2 beliefs are intimately related. For example, it is not true, statistically speaking, that a level iv invariance, or any other level of invariance for that matter, is required in order that perfectly coherent 


\section{ACCEPTED MANUSCRIPT}

comparisons of the mean vectors $\boldsymbol{\mu}_{j}, j=1 . . s$, can be made. Level iv invariance is seen, here, as a necessity because it implies that

$$
\boldsymbol{\mu}_{j}-\boldsymbol{\mu}_{k}=\left(\boldsymbol{\tau}+\boldsymbol{\Lambda} \kappa_{j}\right)-\left(\boldsymbol{\tau}+\boldsymbol{\Lambda} \kappa_{k}\right)=\boldsymbol{\Lambda}\left(\kappa_{j}-\kappa_{k}\right),
$$

and researchers wish to employ inferential knowledge about differences in the $\boldsymbol{\mu}_{j}, j=1 . . s$, to make decisions about differences in the common factor means $\kappa_{j}, j=1 . . s$. But differences in the $\kappa_{j}, j=1 . . s$, are of interest to researchers just because they believe that the $\kappa_{j}$ can be interpreted as means of the same variable (or, as many phrase it (e.g., Wu, Li and Zumbo, 2007), the same construct) distributed in $s$ distinct populations.

Given that both turn out to rest on a concern for the equivalence of the common factor random variables $\xi_{j}, j=1 . . s$, it is, perhaps, not then surprising to encounter, in the literature, blendings of Class 1 and Class 2 beliefs:

"For if one did not know that such measurement invariance obtained, then any differences between means could just as well be interpreted as indicating that different things were measured..." (Horn and McArdle, 1992, p.117) "...the statement of McArdle (1996) that equality of factor loadings should be established before other group comparisons (e.g., mean differences) were considered is worth noting. If not, the psychological constructs being measured may be qualitatively different for the groups being compared" (Colom, Juan-Espinosa, Abad, and Garcia, 2000, p.58).

"...the tests are unbiased with respect to group. This implies that the same constructs are measured in the groups" (Dolan, Roorda, and Wicherts, 2003, p.156). 


\section{ACCEPTED MANUSCRIPT}

"...support for the equality in the last three elements may suggest that the two groups may, in fact, belong to the same population regarding the construct of interest" (Wu, Li, and Zumbo, 2007, p.3)

We are interested in the issue of the implications of factorial invariance for the equivalence of the common factor random variables $\xi_{j}, j=1 . . s$; in particular, the truth value of the proposition that a level iv invariance establishes the equivalence of the $\xi_{j}, j=1 . . s$. It should be a straightforward matter to address this issue, for it requires only that we: a) note various extant senses of random variable equivalence; b) provide a technical characterization of the random variables the equivalence of which is at issue, i.e., $\xi_{j}, j=1 . . s$; and c) deduce implications of the levels of factorial invariance for the equivalence- in the senses thus identified- of these variables.

The historical strangeness of treatments of the issue is, however, evident in failures in respect (a)- wherein, one encounters endemic equivocation over the term equivalent, as it should be applied to random variables that happen to be latent, this equivocation frequently accompanied by a reluctance to invoke standard technical definitions- and in respect (b)wherein, rather than careful mathematical characterization, one is confronted with a widely accepted, nontechnical account of common factor variables (latent variables, more generally) that stretches back into the infancy of the linear factor model (latent variable modeling technologies, more generally), and has since caught hold within, and spread throughout, applied statistics. The nontechnical characterization to which we refer is, in fact, a metaphysics, wherein the common factor variables are portrayed as being unobservable, and the analyzed variables contained within the vectors $\mathbf{X}_{j}$, as observable. ${ }^{\text {iii }}$ 


\section{ACCEPTED MANUSCRIPT}

Our approach will be to return the problem to the technical domain by setting aside the metaphysics in favour of a proper, mathematical, clarification of the $\xi_{j}$. This yields not only a straightforward adjudication of our original issue in terms of standard, technical, senses of random variable equivalence, but, we believe, interesting insights into random variables that happen to be latent, including a technical basis of differentiation between latent and manifest random variables. We begin by providing a brief review of the concepts variable, random variable, and equivalent random variables.

\section{Variables and random variables}

A variable $X$ is a contrast-class $T_{X}$ of properties $\left\{\gamma_{1}, \gamma_{2}, \ldots, \gamma_{t}\right\}$ " ...that are mutually exclusive and jointly exhaustive..." (Rozeboom, 1988, p.212) over a population of objects $\Delta$. A numerical variable $Y$ over $\Delta$ is a pair $\left\{T_{Y}, g_{Y}\right\}$ in which $g_{Y:} T_{Y} \rightarrow \mathfrak{R}$ maps each $\gamma_{k} \in T_{Y}$ into a number; hence, maps each $i \in \Delta$ (which belongs to one and only one $\gamma_{k}$ ) into a number that represents $i$ 's property in $T_{Y}$ (scales $i$ in respect the properties contained in $T_{Y}$ ) (Rozeboom, 1988). Consider an infinite sequence $M_{Y}$ of the act of drawing from population $\Delta$ an $i$ and assigning it to an element of $T_{Y} . M_{Y}$ yields, then, an infinite sequence of mappings of the $i \in \Delta$ into numbers. Let $I_{i(k)}=1$ when $i$ is assigned to $\gamma_{k}$, and $I_{i(k)}=0$ otherwise. In the classical terminology of probability theory, $M_{Y}$ is called a random system if and only if, for $k=1 . . t$,

$\lim _{n \rightarrow \infty}\left(\frac{\sum_{i=1}^{n} I_{i(k)}}{n}\right) \rightarrow p_{k} \in \mathfrak{R}$ (Fraser, 1976). In the event that $M_{Y}$ is a random system: a) $Y$ is called a (numerical) random variable; b) the numbers $p_{k}, k=1 . . t$, are probabilities, over $\Delta$, and in respect 


\section{ACCEPTED MANUSCRIPT}

to random system $M_{Y}$; equivalently, probabilities of random variable $Y$. To clarify the nature of a numerical random variable is to describe, in detail, the elements of the triple $\left\{T_{Y,}, g_{Y}, M_{Y}\right\}$.

Aspects of the triple $\left\{T_{Y}, g_{Y}, M_{Y}\right\}$ are referred to in the highly compressed formalisms of probability theory. Thus, we have the general, textbook definition of the concept random variable.

Definition: random variable. Let $\left(\Omega, A, P_{A}\right)$ be a probability space and let $\left(\Omega^{\prime}, A^{\prime}\right)$ be a measurable space. Then every $A-A^{\prime}$-measurable mapping $Y: \Omega \rightarrow \Omega^{\prime}$ is called a random variable (with values in $\left.\Omega^{\prime}\right)$ (Bauer, 1972).

In the above definition, $\Omega$, called the sample space, is simply a contrast class $T_{Y}, A$ is a $\sigma$ algebra in $\Omega$ [i.e., the subset of the power set of $\Omega$ that is closed under countable unions, intersections and complementation], and $P_{A}$ is a probability measure (informally, the set $\left.\left\{p_{1}, p_{2}, \ldots, p_{t}\right\}\right)$ generated under some random system $M_{Y}$, the nature of which is not specified by $\left(\Omega, A, P_{A}\right)$.

If it happens to be the case that $\Omega^{\prime} \equiv \Re$, then $A^{\prime} \equiv B$ is a Borel algebra, the $A-A^{\prime}-$ measurable mapping is $g_{Y}$, and $Y$ is a numerical random variable. The induced distribution of numerical random variable $Y$ is the triple $\left(\Re, B, P_{B}\right)$, in which $B$ is the induced Borel algebra of $Y$ $\left(B \equiv\left\{W \subset \mathfrak{R} \mid g_{Y}{ }^{-1}(W) \in A\right\}\right)$, and $P_{B}$ is the induced probability measure $P_{B}: B \rightarrow[0,1]\left(P_{B}(\mathrm{~W}) \equiv\right.$ $\left.P_{A}\left(g_{Y}{ }^{-1}(\mathrm{~W})\right), \forall \mathrm{W} \in B\right)$. Finally, letting the set $Z_{y}$ stand for the closed half space $(-\infty, y]$, the distribution function $F_{Y}$ of $Y$ is induced as follows: $F_{Y}(y)=P(Y \leq y) \equiv P_{B}\left(Z_{y}\right)=P_{A}\left(g_{Y}{ }^{-1}\left(Z_{y}\right)\right) \forall y \in \mathfrak{R}$

Though useful for their compactness of expression of certain key notions, the formalisms of probability theory are semantically empty and, hence, insufficient in respect to a clarification of the nature of a numerical random variable. For although they refer to aspects of the triple 


\section{ACCEPTED MANUSCRIPT}

$\left\{T_{Y}, g_{Y}, M_{Y}\right\}$, they are mute on the details. In particular, they are egregiously silent on the issues of: (a) the semantic natures of the elements $\left\{\gamma_{1}, \gamma_{2}, \ldots, \gamma_{t}\right\}$ of $T_{Y}(\Omega$ in the notation of probability theory); (b) the identity of the mapping $g_{Y}$ : $T_{Y} \rightarrow \mathfrak{R}$ (i.e., measurable mapping $Y: \Omega \rightarrow \mathfrak{R}$ ); and (c) the details of the random system under which the probability measure $P_{A}$ (hence, $P_{B}$, hence, $F_{Y}$ ) was generated.

We raise these points because our concern is with the issue of the equivalence of the random variables $\xi_{j}, j=1 . . s$, random variables that happen to be common factors, hence, latent variables, and where one encounters latent variables, characterization is standardly supplanted by empty formalisms that are "explained"- somewhat surprisingly- with reference to an unchallenged, extra-mathematical, metaphysics; or, just as commonly, the metaphysics is cited in defense of the view that, because the random variables in question happen to be latent variables, these variables must elude characterization of the usual, technical, form. A technical issue of random variable equivalence cannot be resolved when set upon such shaky foundations, and our remedy will be precisely to eliminate the metaphysics by insisting upon a specification of $\left\{T_{\xi \xi}, g_{\xi \xi j}\right\}, j=1 . . s$.

\section{The concept equivalent random variables}

There have been invented a number of senses of the concept equivalent random variables, arguably the most important given below, and ordered from strongest to weakest in the sense of material implication (Pfeiffer, 1978; Randles and Wolfe, 1979):

Identical random variables. Let numerical random variables $Y_{l}, l=1 . . p$, be measurable mappings $g_{Y l} \Omega \rightarrow \Omega^{\prime}$ from the same measurable space $(\Omega, A)$. Then the $Y_{l}$ are identical iff for every $\omega \in \Omega$, $g_{Y I}(\omega)=g_{Y 2}(\omega)=\ldots=g_{Y p}(\omega)$. 


\section{ACCEPTED MANUSCRIPT}

wp1 equivalence. Let numerical random variables $Y_{l}, l=1 . . p$, be measurable mappings $g_{Y l}$ : $\Omega \rightarrow \Omega^{\prime}$ from the same measurable space $(\Omega, A)$. Then the $Y_{l}$ are wp1 equivalent iff for $l=1$..p $-1, l^{\prime}$ $=l+1 \ldots p, \mathrm{P}\left(Y_{l}=Y_{l^{\prime}}\right)=1$.

Equal in distribution. Numerical random variables $Y_{l}, l=1 . . p$, are equal in distribution iff $F_{Y I}(y)$ $=F_{Y 2}(y)=\ldots=F_{Y p}(y), \forall y \in \Re$.

Partial Moment Sequence Equivalence. Numerical random variables $Y_{l}, l=1 . . p$, are partially moment sequence equivalent iff, for at least one value of $r=\{1,2,3, \ldots\}, \mathrm{E}\left(Y_{l}^{r}\right)=\mathrm{E}\left(Y_{2}^{r}\right)=\ldots=$ $\mathrm{E}\left(Y_{p}^{r}\right)$

We note that: (a) the strongest two senses of equivalence, but not the weakest two, require that the random variables be defined on the same measurable space; (b) the condition of wp1 equivalence is equivalent to the condition on the pairwise joint distributions of the random variables that, for $l=1 . . p-1, l^{\prime}=l+1 . . p, P\left(Y_{l}=y_{l} \cap Y_{l^{\prime}}=y_{l^{\prime}}\right) \neq 0$ only when $y_{l}=y_{l^{\prime}}$; (c) The condition of complete moment sequence equivalence is identical to the condition of equal in distribution.

\section{An obscuring metaphysics}

Now, to apply these senses of equivalent random variables to the $\xi_{j}, j=1 . . s$, we require a technical characterization of common factor random variables. It is here that we encounter, instead, a metaphysical picture- traceable to the mid-twentieth century influence of empirical realist philosophy on the foundational work on latent variable modeling of, among others, L.L. Thurstone (1947), Paul Lazarsfeld (1959), Frederic Lord and Melvin Novick (1968), and now ubiquitous throughout empirical science and applied statistics- in which the numerical random variables contained within the $\mathbf{X}_{j}$ (manifest variables, in general) are said to be observable, and 


\section{ACCEPTED MANUSCRIPT}

the numerical random variable $\xi_{j}$ (latent variables, in general), unobservable. This is a metaphysical picture because it is neither an empirical or scientific claim, nor a conceptual claim, nor a mathematical claim. It is a claim that has neither been supported by evidence, nor by proof. Most tellingly, it is a claim that could neither be supported by evidence or proof.

It is "supported", instead, by: (a) endemic, illegitimate equatings of concepts, e.g., the concepts random variable, concept, construct, measured property and variants thereof; (b) the drawing of ungrounded and illegitimate analogies with the problems attendant to the perceptual unobservability of material entities that standardly arise within the natural sciences; and (c) loose, figurative language, including the language derived from the resource extraction metaphor that portrays the elements of $\mathbf{X}$ as "tapping into" the latent realms in which the $\xi$ "reside".

The standard yield of a metaphysical picture is incoherence, and this particular picture asserts a category error: being as they are constituted of contrast classes paired with (mapping) functions, numerical variables can be neither observable, nor unobservable. Unlike material entities, contrast classes and mapping functions, hence, numerical variables, are not the right kinds of thing to lie on the continuum whose poles are observable and unobservable.

We need to move beyond this obscuring metaphysics, and derive a clarification of the random variables $\xi_{j}, j=1 . . s$, this clarification involving specifications of the $\left\{T_{\xi j}, g_{\xi j}\right\}$. This aim, it turns out, benefits greatly from an initial setting down of a technical standard of differentiation between latent and manifest random variables, a task to which we now turn.

\section{A technical standard of differentiation between manifest and latent variables}

Let it be the case that, within a population $\Delta$ of objects under study, $\mathbf{X}$ is representable by the unidimensional, linear factor model; i.e., $\mathbf{X}=\boldsymbol{\tau}+\boldsymbol{\Lambda} \xi+\boldsymbol{\delta}$, in which $\mathrm{E}(\boldsymbol{\delta})=\mathbf{0}, \mathrm{C}(\boldsymbol{\delta})=\boldsymbol{\Theta}_{\boldsymbol{\delta}}$, 


\section{ACCEPTED MANUSCRIPT}

$\mathrm{E}(\xi)=0, \mathrm{~V}(\xi)=1$, and $\mathrm{C}(\xi, \boldsymbol{\delta})=\mathbf{0}$. We require a technical (non-metaphysical) standard of differentiation between the manifest variables contained in $\mathbf{X}$ and the latent variable $\xi$. Note, first, that for each element of $\mathbf{X}$, but not for $\xi$, the pair $\{T, g\}$ must be specified prior to carrying out a factor analysis. That the pairs $\left\{T_{l}, g_{l}\right\}, l=1 . . p$, but not the pair $\left\{T_{\xi}, g_{\xi}\right\}$, must be specified antecedently is manifested in the fact that the data that is factor analyzed in a factor analysis is a sample drawn from $\Delta$ on $\mathbf{X}$, but not on $\xi$.

Thus, a researcher specifies prior to analysis that the elements $\left\{\gamma_{1 l}, \gamma_{2 l}, \ldots, \gamma_{t l}\right\}$ of $T_{l}$ are, for example, the $t_{l}$ response options of item $l$ of some particular test, that these options will be "coded" as specified by the functions $g_{l}: T_{l} \rightarrow \mathfrak{R}, l=1 . . p$, and that $\mathbf{X}$ will be made random under the idealization of repeated, in vaccuo, (random) sampling from a particular population $\Delta$ of entities under study (see Holland, 1990). The underlying space of $\mathbf{X}$ is, then, $\left(\Omega_{\mathbf{X}}, A_{\mathbf{X}}, P_{\mathbf{X}}\right)$ in which $\Omega_{\mathbf{X}} \equiv \stackrel{p}{\gtrless}_{l=1}^{\otimes} T_{l}$ and in which $P_{\mathbf{X}}$ is generated under repeated, in vaccuo, sampling from particular population $\Delta$. The induced distribution of $\mathbf{X}$, i.e., under measurable mapping $\mathbf{g}: \Omega_{\mathbf{X}} \rightarrow$ $\mathfrak{R}^{p}$, is $\left(\mathfrak{R}^{p}, B_{g}, P_{g}\right)$. Letting $z_{l}$ stand for the closed half space $(-\infty, x]$ and $Z_{g}=\stackrel{p}{l}_{l=1}^{p} z_{l}$, the induced distribution function $F_{\mathbf{X}}(X)=P(\mathbf{X} \leq X) \equiv P_{g}\left(Z_{g}\right)=P_{\mathbf{X}}\left(g^{-1}\left(Z_{g}\right)\right) \forall X \in \mathfrak{R}^{p}$. In testing hypotheses bearing on the parameters $\left\{\boldsymbol{\Lambda}, \boldsymbol{\tau}, \boldsymbol{\Theta}_{\delta}, \kappa\right\}, F_{\mathbf{X}}(X)$, which is determined by $P_{g}$, is commonly taken as being adequately represented by some continuous $p$-dimensional distribution function $\tilde{F}$.

What, then, of the numerical random variable $\xi$ ? How is its nature settled, or, in other words, on what basis is $\left\{T_{\xi}, g_{\xi}\right\}$ specified? In contradistinction to the elements of $\mathbf{X}$, the nature of $\xi$ is determined, post-hoc (i.e., under the condition that the unidimensional, linear factor model happens to represent $\mathbf{X}$ in a population $\Delta$ ), by the totality of constraints placed upon it by the 


\section{ACCEPTED MANUSCRIPT}

unidimensional, linear factor model. Let these constraints be the elements of set $C_{u l f}$. Then: a) $C_{u l f}$ can be thought of as a generating recipe or construction formula; $\mathrm{b}$ ) the symbol $\xi$ stands for any random variable that satisfies $C_{u l f}$, hence, for those random variables generated in conformity to $C_{u l f}$; c) the label "common factor to $\mathbf{X}$ " can be legitimately ascribed to just those random variables generated in conformity to $C_{u l f}$.

The latent variables referred to in the defining equations of any and all latent variable models are simply generated random variables. Let: $M$ be any $t$-dimensional latent variable model; $\mathbf{X}$ be the $p$ "manifest" random, and $\xi$, the $t$ "latent" random, variables, referred to in the defining equations of $M$; and $C_{M} \equiv\left\{c_{1}, c_{2}, \ldots\right\}$ be the totality of $M$-specific constraints that $\xi$ must satisfy when $M$ represents $\mathbf{X}$. Then $\boldsymbol{\xi}$ is a vector of $t$ generated random variables, or, in other words, random variables for which $\left\{T_{\xi l}, g_{\xi l}\right\}, l=1 . . t$, are fixed post-hoc (i.e., under the condition that $M$ happens to represent $\mathbf{X}$ in a population $\Delta$ ) by $C_{M}$.

Because the traditional manifest variable and latent variable terminology is, now, inextricably linked to the insidious metaphysical picture under which $\mathbf{X}$ and $\boldsymbol{\xi}$ are misrepresented as being observable and unobservable, respectively, we recommend replacing these terms with the, more technical, and explanatory, terms input- (for each of the elements of $\mathbf{X}$; those random variables realizations on which constitute the data of a latent variable analysis) and generated (for each of the elements of $\xi$; those random variables the natures of which are fixed by model specific constraints) random variable. ${ }^{\text {iv }}$

Generated random variables are, of course, commonplace within statistics. Let $V$ be any statistical model; $\mathbf{X}$ be the set of $p$ input random variables (variables on which realizations are taken prior to analysis), and $\boldsymbol{\eta}, t$ generated random variables (variables for which $\left\{T_{\eta l}, g_{\eta l}\right\}, l=1 . . t$, 


\section{ACCEPTED MANUSCRIPT}

are fixed post-hoc by a set of $V$-specific constraints $C_{V}$, under condition that $V$ represents $\mathbf{X}$ in a population $\Delta$ ), referred to in the defining equations of $V$. Then, it is useful to distinguish between models $V$ for which $\boldsymbol{\eta}$ is a function of $\mathbf{X}$ (e.g., component models, canonical correlation models, linear and nonlinear regression models of various sorts) and those for which $\boldsymbol{\eta}$ is not a function of $\mathbf{X}$ (the latent variable models).

In respect to a generated random variable $\eta$ that appears in the defining equations of a statistical model $V$, be it either a function of $\mathbf{X}$, or not (examples of this latter type, the variables $\xi_{j}, j=1 . . s$, the equivalence of which is the central question of this paper), certain fundamental issues arise. Existence. Does there (always/never/only given satisfaction of certain conditions) exist a $\eta$ that satisfies model specified constraints $C_{V}$ ? If only given the satisfaction of certain conditions on the distribution of $\mathbf{X}$, what are these conditions? Cardinality. If, for a given $\mathbf{X}$, there does exist one or more $\eta$, each of which satisfies $C_{V}$, how many such $\eta$ do in fact exist? Deduction of $\left\{T_{\eta}, g_{\eta}\right\}$. A numerical random variable is one part contrast class and one part mapping function. If, for a given $\mathbf{X}$, distributed over a given population $\Delta$, there exists one or more $\eta$, each of which satisfies $C_{V}$, what are the elements of contrast class(es) $T_{\eta}$ and the identity of the function(s) $g_{\eta}$ that maps $i \in \Delta_{j}$ onto $\Re$. Can (the) $g_{\eta}$ be deduced on the basis of $C_{V}$ ?

Contingent properties of $\eta$. If, for a given $\mathbf{X}$, there exists one or more $\eta$, each of which satisfies $C_{V}$, the most fundamental issue here is the issue of the induced distribution(s) of $\eta$. Example (First principal component variable). Let $\mathbf{X}=\eta_{1}+\boldsymbol{\varepsilon}$, in which $\mathbf{X}$ is a $p$-vector of input random variables and $\eta_{1}$ is the first principal component variable to $\mathbf{X}$. Then $\eta_{1}$ is a generated random variable, and the generating recipe is $C_{p c l} \equiv\left\{c_{1}, c_{2}\right\}$, in which $c_{1}->$ " $\eta_{1}=\mathbf{t}$ 'X (i.e., $\eta_{1}$ is a linear function of $\mathbf{X})$ " and $c_{2}->$ "V $\left(\eta_{1}\right)=\mathbf{t}^{\prime} \Sigma \mathbf{t}>0$ is a maximum over all normed $\left(\mathbf{t}^{\prime} \mathbf{t}=1\right) p$ - 


\section{ACCEPTED MANUSCRIPT}

vectors of real constants". Existence. Because $\Sigma$ is gramian, $\eta_{1}$ always exists. Cardinality. $\eta_{1}$ is unique if and only if $\lambda_{1}>\lambda_{r}, r=2 . . p$, in which $\lambda_{s}$ is the $s$ th in the ordered (from largest to smallest) set of $p$ eigenvalues of $\Sigma$. Deduction of $g_{\eta 1}$. As is well known (e.g., Mardia, Kent, and Bibby, 1979), $\eta_{1}$ satisfies $C_{p c l}$ if and only if $\mathbf{t}$ is the eigenvector of $\Sigma$, say, $\mathbf{v}_{1}$, associated with the largest eigenvalue of $\Sigma$, say, $\lambda_{1}$, from which it follows that $g_{\eta 1}(\mathbf{X})=\mathbf{v}_{1}{ }^{\prime} \mathbf{X}\left(\right.$ with $\left.\mathrm{V}\left(\eta_{1}\right)=\mathrm{V}\left(\mathbf{v}_{1}{ }^{\prime} \Sigma \mathbf{v}_{1}\right)=\lambda_{1}\right)$.

\section{The nature of the common factor random variable}

The generating recipe of the unidimensional, linear factor model is as follows:

$\xi$ is a common factor to input random $p$-vector $\mathbf{X}$ if and only if:

ulf1) $\mathrm{E}(\xi)=0$

ulf2) $\mathrm{E}\left(\xi^{2}\right)=1$;

ulf3) the vector of residuals of the linear regression of $\mathbf{X}$ on $\xi, \mathbf{X}-\boldsymbol{\tau}-\boldsymbol{\Lambda} \xi$, has a

covariance matrix $\mathrm{C}(\mathbf{X}-\boldsymbol{\tau}-\boldsymbol{\Lambda} \xi)$ that is diagonal and positive definite.

In other words, $C_{u l f} \equiv\{$ ulf1,ulf2,ulf3 $\}$ and we have the following, well known, answers to the questions of existence, cardinality, and the like.

Existence. Let input random $p$-vector $\mathbf{X}$ be distributed over a population $\Delta$. As is well known (Guttman, 1955; Wilson, 1928), there exists at least one random variable $\xi$ that satisfies $C_{u l f}$ if and only if there exists a vector $\boldsymbol{\Lambda}$ of real numbers and a diagonal and positive definite matrix $\boldsymbol{\Theta}_{\delta}$ such that $\mathrm{C}(\mathbf{X})=\Sigma=\mathbf{\Lambda}^{\prime}+\boldsymbol{\Theta}_{\delta}$.

Deduction of $g_{\xi}$. Given satisfaction of the condition of existence, $g_{\xi}=g_{\xi}(\mathbf{X})=\mathbf{\Lambda} \mathbf{\Sigma}^{-1} \mathbf{X}+w^{1 / 2} s *$, in which $w=\left(1-\boldsymbol{\Lambda}^{\prime} \boldsymbol{\Sigma}^{-1} \boldsymbol{\Lambda}\right)$ and $s *$ is any random variable for which $\mathrm{C}(s * \mathbf{X})=\mathbf{0}, \mathrm{E}\left(s_{*}\right)=0$, and $\mathrm{V}(s *)=1\left(\right.$ Guttman, 1955; Kestelman, 1952; Piaggio, 1931). ${ }^{\mathrm{V}}$ 


\section{ACCEPTED MANUSCRIPT}

Cardinality. Let set $L_{u l f}$ contain all random variables $\xi$ that satisfy $C_{u l f}$. Because there can be constructed an infinity of distinct random variables $s *^{(m)}, m=\{1,2, \ldots\}$, for which $\mathrm{C}\left(s^{(m)}, \mathbf{X}\right)=\mathbf{0}$, $\mathrm{E}\left(s *^{(m)}\right)=0$, and $\mathrm{V}\left(s *{ }^{(m)}\right)=1$, it follows that, given satisfaction of the condition of existence, there exists a countable infinity of random variables $\xi$ that satisfy $C_{u l f}$; i.e., $\operatorname{Card}\left(L_{u l f}\right)=\infty($ Guttman, 1955; Piaggio, 1931; Wilson, 1928). Each element of $L_{u l f}$ is designated by the particular $s *^{(m)}$ to which it is tied. Thus, $\xi_{m}$ is just that element of $L_{u l f}$ for which $g_{\xi m}(\mathbf{X})=\mathbf{\Lambda}^{\prime} \boldsymbol{\Sigma}^{-1} \mathbf{X}+w^{1 / 2} \boldsymbol{S *}^{(m)}$. Contingent Properties. The symbol $\xi$ stands for the countable infinity of elements $\xi_{m}$ of $L_{u l f}$. To consider contingent properties of $\xi$ is, therefore, to consider contingent properties of the $\xi_{m} \in L_{u l f}$.

Induced Distributions. The underlying space of $\xi_{m},\left(\Omega_{\mathrm{X}, s^{*}(m)}, B_{\mathrm{X}, s^{*}(m)}, P_{\mathrm{X}, s^{*}(m)}\right)$, is generated by a compound experiment consisting of two component experiments. In particular, under repeated, in vaccuo, sampling from a population $\Delta$ of objects under study, to each $i$ sampled assign: i) $p$ scores in accordance with $\boldsymbol{g}: \Omega_{\mathrm{X}} \rightarrow \mathfrak{R}^{P}$; ii) a $(p+1) t h$ score that is a realization on a random variable $s *{ }^{(m)}$ chosen so as to have the properties $\mathrm{C}\left(s *^{(m)}, \mathbf{X}\right)=\mathbf{0}, \mathrm{E}\left(s *^{(m)}\right)=0$, and $\mathrm{V}\left(s *^{(m)}\right)$ $=1$. Thus: $\Omega_{\mathrm{X}, s^{*}(m)} \equiv \mathfrak{R}^{P} \otimes \Omega_{s^{*}(m)}$, in which $\Omega_{s^{*}(m)}$ is the sample space of $s *^{(m)} ; B_{\mathrm{X}, s^{*}(m)}$ is the Borel algebra; and $P_{\mathrm{X}, s^{*}(m)}$ is the probability measure (which determines the joint distribution function of $\mathbf{X}$ and $\left.s *^{(m)}\right)$.

The induced distribution of $\xi_{m}$ is, then, the triple ( $\left.\mathfrak{R}, B_{\xi m}, P_{\xi m}\right)$, in which $B_{\xi m}$ is the induced Borel algebra $\left(B_{\xi m} \equiv\left\{W \subset \Re \mid g_{\xi m}{ }^{-1}(W) \in B_{\mathrm{X}, s^{*}(m)}\right\}\right)$ and $P_{\xi m}$ is the induced probability measure $P_{\xi m}: B_{\xi m} \rightarrow[0,1]\left(P_{\xi m}(W)=P_{\mathrm{X}, *^{*}(m)}\left(g_{\xi m}^{-1}(W)\right), \forall W \in B_{\xi m}\right)$. The induced distribution function, $F_{\xi m}(y)=P\left(\xi_{m} \leq y\right) \equiv P_{\xi m}(Z)=P_{\mathrm{X}, *^{*}(m)}\left(g_{\xi m}^{-1}(Z)\right) \forall y \in \mathfrak{R}$.

Question of equivalence of $\xi_{m} \in L_{u l f}$. A sample space is simply a contrast class, and any $i \in \Delta$ has one and only one property in a contrast class. Each $s_{*}^{(m)}$ assigns each $i \in \Delta$ to a 


\section{ACCEPTED MANUSCRIPT}

different property. Thus, it follows that there is a distinct sample space $\Omega_{s^{*}(m)}$ for each $s *{ }^{(m)}$.

Consequently, each element $\xi_{m}$ of $L_{u l f}$ is defined on a distinct measurable space, and it is concluded that the elements of $L_{u l f}$ are neither identical, nor wp1-equivalent random variables. Moreover, because the $s *^{(m)}$ are restricted by only weak, second-order, moments constraints, there is no necessity that the $P_{\mathrm{X}, s^{*}(m)}$ (hence, the $P_{\xi m}$, hence, the $F_{\xi m}$ ) be identical over the elements $\check{\xi}_{m}$ of $L_{u l f}$. Consequently, there is no necessity that the $\xi_{m} \in L_{u l f}$ be equal in distribution. In fact, the only sense of random variable equivalence that the elements $\xi_{m}$ of $L_{u l f}$ satisfy is that of partial moment sequence equivalence, this a consequence of the generating recipe of linear factor analysis, which requires that each $\xi_{m}$ satisfies (ulf1) and (ulf2).

Issues of mean square similarity of $\xi_{m} \in L_{u l f}$. Although elements $\xi_{m}$ of $L_{u l f}$ are equivalent only in the virtually trivial sense of partial moment sequence equivalence, it may still be asked, more generally, how similar they are in a mean-squared difference sense. This issue may be thought of as the issue of the similarity of the infinity of distinct mappings $g_{\xi m}(i)$ contained within $L_{u l f}$ of the $i \in \Delta$ into numbers that represent the properties of the $i$ in the $T_{\xi m}$. The following results provide some insight in respect this issue. For $\left\{\xi_{m}, \xi_{m^{\prime}}\right\} \in L_{u l f}($ Guttman, 1955):

$$
\rho\left(\xi_{m}, \xi_{m^{\prime}}\right)=\boldsymbol{\Lambda} \boldsymbol{\Sigma}^{-1} \boldsymbol{\Lambda}+w \rho\left(s_{*_{m}}, s_{*^{\prime}}\right) \text {. }
$$

$$
\boldsymbol{\Lambda} \boldsymbol{\Sigma}^{-1} \boldsymbol{\Lambda} \geq \rho\left(\xi_{m}, \xi_{m^{\prime}}\right) \geq 2 \boldsymbol{\Lambda} \boldsymbol{\Sigma}^{-1} \boldsymbol{\Lambda}-1 \text { (6) }
$$

That is to say, even though the elements of $L_{u l f}$ are all common factors to $\mathbf{X}$, their exist pairs $\left\{\xi_{m}, \xi_{m^{\prime}}\right\}$ whose Pearson Product Moment Correlations are as low as $2 \boldsymbol{\Lambda} \boldsymbol{\Sigma}^{-1} \boldsymbol{\Lambda}-1$, a quantity that is negative when $\boldsymbol{\Lambda} \boldsymbol{\Sigma}^{-1} \boldsymbol{\Lambda}<$.5. For arbitrary external random variable $Y$ and $\xi_{m} \in L_{u l f}$ (Steiger, $1979)^{\text {vi }}$ : 


\section{ACCEPTED MANUSCRIPT}

$$
\begin{aligned}
& \rho\left(\xi_{m}, Y\right)=\mathbf{\Lambda} \boldsymbol{\Sigma}^{-1} \sigma_{\mathbf{X}, Y}+w^{1 / 2} \sigma_{s^{*} m, Y} ;(7) \\
& \mathbf{\Lambda} \boldsymbol{\Sigma}^{-1} \sigma_{\mathbf{X}, Y}+\left(1-R_{\mathbf{X} . Y}^{2}\right)^{1 / 2} w^{1 / 2} \geq \rho\left(\xi_{m}, Y\right) \geq \mathbf{\Lambda} \boldsymbol{\Sigma}^{-1} \sigma_{\mathbf{X}, Y}-\left(1-R_{\mathbf{X} . Y}^{2}\right)^{1 / 2} w^{1 / 2}
\end{aligned}
$$

That is to say, even though the elements of $L_{u l f}$ are all common factor to $\mathbf{X}$, they will not have identical Pearson Product Moment Correlations with an arbitrary variable $Y$; the range of the correlations with $Y$ over $L_{u l f}$ is equal to $2\left(1-R^{2} \mathbf{X} . Y\right)^{1 / 2} w^{1 / 2}$. It may be concluded, then, that even in the much weaker sense of mean-square similarity, the elements of $L_{u l f}$ need have little resemblance to each other.

Asymptotic equivalence of $\xi_{m} \in L_{u l f}$ and first principal component variable, $\eta_{1}$. Both any of the elements $\xi_{m}$ of $L_{u l f}$ and the first principal component variable to $\mathbf{X}, \eta_{1}$, are generated random variables. The former exists if and only if, for a given population $\Delta$, it happens to be the case that there exists a vector $\boldsymbol{\Lambda}$ of real numbers and a diagonal and positive definite matrix $\boldsymbol{\Theta}_{\boldsymbol{\delta}}$ such that $\Sigma=\mathbf{\Lambda} \mathbf{\Lambda}^{\prime}+\boldsymbol{\Theta}_{\delta}$. The latter always exists. The former, if it exists, is not, for finite $p, a$ function of $\mathbf{X}$; the latter is.

However, under certain conditions, $\xi_{m}$ and $\eta_{1}$ are asymptotically, as $p \rightarrow \infty$, equivalent (Schneeweiss, 1989; Bentler and Kano, 1990); this suggesting, once again, the absurdity of putting forth observability and unobservability as the basis for distinguishing between $\xi_{m}$ and $\eta_{1}$; more generally, the absurdity of ascribing to latent variables the property of unobservability. The two generated variables $\xi_{m}$ and $\eta_{1}$ are variables, hence, are neither observable, nor unobservable. They are, instead, characterized, and distinguishable, on the basis of their generating recipes and contingent properties.

Let there be a large population of variables $D$, and a sequence of sets of variables $\left\{S_{3}, S_{4}, \ldots, S_{k}\right\}, S_{3}$ formed by drawing a random sample of 3 variables from D, $S_{4}$ formed by 


\section{ACCEPTED MANUSCRIPT}

augmenting $S_{3}$ with a randomly sampled fourth variable, and so on. Let it be the case that each set $S_{j}$ is representable by the unidimensional, linear factor model; i.e., $X_{(j)}=\Lambda_{(j)} \xi_{(j)}+\Psi_{(j)}^{1 / 2} \boldsymbol{\delta}_{(j)}$, j=3. $\infty$, in which $\Psi_{(j)}$ is diagonal and positive definite, $\mathrm{V}\left(\xi_{(j)}\right)=1, \mathrm{C}\left(\boldsymbol{\delta}_{(j)}\right)=\mathrm{I}$, and $\mathrm{C}\left(\xi_{(j)}, \boldsymbol{\delta}_{(j)}\right)=\mathbf{0}$, in consequence of which, $\Sigma_{(j)}=\Lambda_{(j)} \Lambda_{(j)}{ }^{\prime}+\Psi_{(j)}, j=3 . . \infty$. The idea is that the sets $S_{j}$ remain representable by the unidimensional linear factor model as $j$ becomes progressively larger.

Consider, also, the sequence $\Sigma_{(j)} \mathbf{v}_{1(j)}=\lambda_{1(j)} \mathbf{v}_{1(j)}, \mathbf{v}_{1(j)} \mathbf{v}_{1(j)}=1$, in which $\mathbf{v}_{1(j)}$ and $\lambda_{1(j)}$ are the first eigenvector and eigenvalue, respectively, of $\Sigma_{(j)}$. The variable $\eta_{1(j)}=\mathbf{v}_{1(j)}{ }^{\prime} \mathrm{X}_{(j)}$ is the first principal component of the distribution of $S_{j}$.

If, as $j \rightarrow \infty, \Lambda_{(j)}{ }^{\prime} \Lambda_{(j)} \rightarrow \infty$ and there exists a real number $\zeta_{0}>0$ such that, for all $j$ and $k$, $\zeta_{0}>\zeta_{k k(j)}$, in which $\zeta_{k k(j)}$ is the $k k$ th element of $\Psi_{(j)}$, then $\rho\left(\eta_{1(j)}, \xi_{(j)}\right) \rightarrow 1$. Proof. Because $\xi_{(j) m}=$ $\Lambda_{(j)}{ }^{\prime} \Sigma_{(j)}{ }^{-1} \mathrm{X}_{(j)}+w_{(j)}{ }^{1 / 2} S *{ }^{(j, m)}$ are the elements of set $L_{u l f j j}$, i.e., are the common factors to $\mathrm{X}_{(j)}$, it follows that $\rho\left(\eta_{1(j)}, \xi_{(j)}\right)=\rho\left(\mathbf{v}_{1(j)}{ }^{\prime} \mathbf{X}_{(j)}, \boldsymbol{\Lambda}_{(j)}{ }^{\prime} \Sigma_{(j)}{ }^{-1} \mathbf{X}_{(j)}+w_{(j)}{ }^{1 / 2} S{ }^{(j, m)}\right)=\frac{\left(\mathbf{v}_{1(j)}{ }^{\prime} \boldsymbol{\Lambda}_{(j)}\right)}{\sqrt{\lambda_{1(j)}}}$. Now, $\Sigma_{(j)} \mathbf{v}_{1(j)}=\left(\Lambda_{(j)} \Lambda_{(j)}{ }^{\prime}+\Psi_{(j)}\right) \mathbf{v}_{1(j)}$, from which it follows that $\mathbf{v}_{1(j)}{ }^{\prime} \Sigma_{(j)} \mathbf{v}_{1(j)}=\left(\mathbf{v}_{1(j)} \Lambda_{(j)}\right)^{2}+\mathbf{v}_{1(j)}{ }^{\prime} \Psi_{(j)} \mathbf{v}_{1(j)}$. Thus, $\lambda_{1(j)}-\mathbf{v}_{1(j)} \Psi_{(j)} \mathbf{v}_{1(j)}=\left(\mathbf{v}_{1(j)} \Lambda_{(j)}\right)^{2}=\lambda_{1(j)} \rho\left(\eta_{1(j)}, \xi_{(j)}\right)^{2}$. Under condition that there exists a real number $\zeta_{0}>0$ such that, for all $j$ and $k, \zeta_{0}>\zeta_{k k(j)}$, and the fact that $\mathbf{v}_{1(j)} \mathbf{v}_{1(j)}=1$, it follows, finally, that $\rho\left(\eta_{1(j)}, \xi_{(j)}\right)^{2}>\left(1-\frac{\zeta_{0}}{\lambda_{1(j)}}\right)$. Because, as $j \rightarrow \infty, \lambda_{1(j)} \rightarrow \infty$, it follows that $\rho\left(\eta_{1(j)}, \xi_{(j)}\right)^{2} \rightarrow 1$.

\section{Implications of factorial invariance for equivalence of the $\xi_{j}$}

Consider, once again, the situation in which each of $\mathbf{X}_{j}, j=1 . . s$, is representable by the unidimensional, linear factor model, i.e., for each, representation (1) holds. What, then, does the level of factorial invariance that holds over $\Delta_{j}, j=1 . . s$, imply about the equivalence of the $\xi_{j}, j=$ 


\section{ACCEPTED MANUSCRIPT}

$1 . . s ?$ We note, immediately, that, when purged of metaphysical accompaniment, the issue turns out to be more complicated than it initially appeared, for the reason that the symbols $\xi_{j}, j=1 . . s$, actually stand for the countable infinities of elements $\xi_{m(j)}$ of the sets $L_{u l f(j)}, j=1$..s. The issue must, then, be rephrased as follows: What does the level of factorial invariance that holds over $\Delta_{j}, j=1 . . s$, imply about the equivalence of any pair of elements $\left\{\xi_{m(j)}, \xi_{m^{\prime}\left(j^{\prime}\right)}\right\}, j \neq j^{\prime}$, i.e., random variables belonging to distinct $L_{u l f(j)}$ ?

To come to a conclusion, we need only consider the most restrictive case, level iv or strict factorial invariance. Level iv invariance implies that, for $j=1 . . s$ :

$$
\begin{aligned}
& 1+w_{j}=\boldsymbol{\Lambda}_{j} \boldsymbol{\Sigma}_{j}^{-1} \boldsymbol{\Lambda}_{j}=\mathbf{\Lambda} \boldsymbol{\Sigma}^{-1} \boldsymbol{\Lambda}=1+w ;(9) \\
& g_{\xi m(j)}\left(\mathbf{X}_{j}\right)=\boldsymbol{\Lambda}_{j}{ }^{\prime} \boldsymbol{\Sigma}_{j}{ }^{-1} \mathbf{X}_{j}+w_{j}{ }^{1 / 2} \boldsymbol{s}_{j^{*}}{ }^{(m)}=\boldsymbol{\Lambda}^{\prime} \boldsymbol{\Sigma}^{-1} \mathbf{X}_{j}+w^{1 / 2} \boldsymbol{s}_{j^{*}}{ }^{(m)}
\end{aligned}
$$

We see, then, that, under level iv invariance, the functions that map each $i \in \Delta_{j}$ into a

number are identical over all of the random variables contained in $\bigcup_{j=1}^{s} L_{u l f(j)}$. Hence, under level iv invariance, precisely the same conclusions in respect to the issue of random variable equivalence hold for any pair of elements $\left\{\xi_{m(j)}, \xi_{m^{\prime}\left(j^{\prime}\right)}\right\}$ belonging to $\operatorname{distinct} L_{u l f(j)}$, as were shown to hold for any pair belonging to the same $L_{u l f(j)}$.

Thus, we may conclude as follows: (a) because $\xi_{m(j)}$ and $\xi_{m^{\prime}\left(j^{\prime}\right)}$ are defined on distinct measurable spaces, they are neither identical, nor wpl-equivalent random variables; (b) because they are induced by $P_{\mathrm{Xj}, s j^{*}(m)}$ and $P_{\mathrm{Xj}^{\prime}, s j^{\prime *}\left(m^{\prime}\right)}$, respectively- which, being the joint distributions of $\mathbf{X}_{j}$ defined over different populations and distinct $s *{ }^{(m)}$, need not be equivalent$F_{\xi m(j)}$ and $F_{\xi m^{\prime}\left(j^{\prime}\right)}$ need not be identical, and, consequently, $\xi_{m(j)}$ and $\xi_{m^{\prime}\left(j^{\prime}\right)}$ need not be equal in 


\section{ACCEPTED MANUSCRIPT}

distribution; (c) because they must all satisfy (ulf1) and (ulf2), the elements of $\bigcup_{j=1}^{s} L_{u l f(j)}$ are

partially moment sequence equivalent. This trivial sense of equivalence is not, however, brought about by level iv factorial invariance, but, rather, by $C_{u l f}$ itself.

\section{Tacit nontechnical senses of equivalence admitted under the metaphysics}

What is the explanation for the existence of the false belief that a determination of the level of factorial invariance that holds over a set of $k$ populations $\Delta_{j}, j=1 . . s$, is central to ascertaining whether or not the common factor random variables $\xi_{j}, j=1 . . s$, are equivalent? A candidate explanation, one suggested originally by Guttman (1977), is that individuals who hold this belief are simply guilty of the (minor) cognitive error of conflating the parameters of representation (1) with the common factor random variables that are symbolized in these representations (see Heene, 2008, for an illustration of this parameter/random variable conflation).

Though there may be something to this, it seems to us- especially, in light of the number of otherwise quantitatively sophisticated individuals who subscribe to this view- that there is something else, here, at work. We suggest that what we are seeing is yet another manifestation of the powerful, insidious, influence of metaphysics upon technical, statistical, work, and believe the illogic in play to be describable as follows: a) under the illegitimate ascription of the property of unobservability to common factor random variables, these variables tacitly come to be treated as entities, processes, and other natural phenomena (those things to which predication of unobservability is legitimate); b) under the identification of common factor random variables with entities, processes, and other natural phenomena, technical senses of random variable 


\section{ACCEPTED MANUSCRIPT}

equivalence are tacitly replaced by (nontechnical) notions of identical or equivalent or similar phenomena; c) finally, the levels of factorial invariance are seen, tacitly, and in an unspecified, nontechnical, fashion, as constituting different degrees of evidence that these phenomena are identical or equivalent or similar. 


\section{ACCEPTED MANUSCRIPT}

\section{References}

Bauer, H. (1972). Probability Theory and Elements of Measure Theory. New York: Holt, Rinehart and Winston.

Bentler, P.M., \& Kano, Y. (1990). On the equivalence of factors and components. Multivariate Behavioural Research, 25(1), 67-74.

Byrne, B. M., \& Watkins, D. (2003). The issue of measurement invariance revisited. Journal of Cross-Cultural Psychology, 34(2), 155-175.

Colom, R., Juan-Espinosa, M., Abad, F., \& Garcia, L. F. (2000). Negligible Sex Differences in General Intelligence. Intelligence, 28(1), 57-68.

Dolan, C. V., Roorda, W., \& Wicherts, J. M. (2004). Two failures of Spearman's hypothesis: The GATB in Holland and the JAT in South Africa. Intelligence, 32(2), 155-173.

Fraser, D.A.S. (1976). Probability and statistics: Theory and applications. Toronto: DAI Press.

Guttman, L. (1955). The determinacy of factor score matrices with implications for five other basic problems of common-factor theory. British Journal of Statistical Psychology, 8, 6581.

Guttman, L. (1977). What is not what in statistics. The Statistican, 26(2), 81-107.

Holland, P. W. (1990) “On the Sampling Theory Foundations of Item Response Theory Models." Psychometrika 55(4), 577-601.

Heene, M. (2008). A Rejoinder to Mackintosh and some Remarks on the Concept of General Intelligence. arXiv: Archive for Electronic Preprints of Scientific Papers. Retrieved from http://arxiv.org/ftp/arxiv/papers/0808/0808.2343.pdf. 


\section{ACCEPTED MANUSCRIPT}

Horn, J. L., \& McArdle, J. J. (1992). A Practical and Theoretical Guide to Measurement Invariance in Aging Research. Experimental Aging Research, 18(3-4), 117-144.

Joreskog, K. (1971). Simultaneous factor analysis in several populations. Psychometrika, 36(4), 409-426.

Kestelman, H. (1952). The Fundamental Equation of Factor Analysis. British Journal of Psychology, 5, 1-6.

Lazarsfeld, P. F. (1959). Latent structure analysis. In S. Koch (Ed.), Psychology: A Study of a Science, Vol. 3. New York: McGraw-Hill.

Lord, F., \& Novick, M. (1968). Statistical Theories of Mental Test Scores. London: Addison-Wesley Publishing Company.

Lubke, G. H., Dolan, C. V., Kelderman, H., \& Mellenbergh, G. J. (2003). Weak measurement invariance with respect to unmeasured variables: An implication of strict factorial invariance. British Journal of Mathematical \& Statistical Psychology, 56, 231-248.

Maraun, M. (2003). Myths and Confusions: Psychometrics and the Latent Variable Model. Retrieved from http://www.sfu.ca/ maraun/Mikes\%20page\%20Myths\%20and\%20Confusions.html.

Mardia, K., Kent, J., \& Bibby, J. (1979). Multivariate Analysis. London: Academic Press, Inc.

McDonald, R. (1977). The indeterminacy of components and the definition of common factors. British Journal of Mathematical \& Statistical Psychology, 30, 165-176.

Meredith, W. (1964). Notes on factorial invariance. Psychometrika, 29(2), 177-185.

Meredith, W. (1993). Measurement invariance, factor analysis and factorial invariance. Psychometrika, 58(4), 525-543. 


\section{ACCEPTED MANUSCRIPT}

Meredith, W., \& Teresi, J. A. (2006). An essay on measurement and factorial invariance. Medical Care, 44(11), 69-77.

Millsap, R. E. (1997). Invariance in measurement and prediction: Their relationship in the singlefactor case. Psychological Methods, 2(3), 248-260.

Millsap, R. E., \& Kwok, O. M. (2004). Evaluating the impact of partial factorial invariance on selection in two populations. Psychological Methods, 9(1), 93-115.

Millsap, R. E., \& Yun-Tein, J. (2004). Assessing factorial invariance in ordered-categorical measures. Multivariate Behavioral Research, 39(3), 479-515.

Mulaik, S. (1990). Blurring the distinctions between component analysis and common factor analysis. Multivariate Behavioral Research, 25 (1), 53-59.

Pfeiffer, P.E. (1978). Concepts of probability theory. London: Dover.

Piaggio, H. T. H. (1931). The general factor in Spearman's theory of intelligence. Nature, 127, $56-57$.

Randles, R.H., and Wolfe, D.A. (1979). Introduction to the theory of nonparametric statistics. New York: Wiley.

Rozeboom, W. (1988). Factor indeterminacy: The saga continues. British Journal of Mathematical and Statistical Psychology, 41, 209-226.

Schneeweiss, H. (1989, June). Models with latent variables. Paper presented at Joint Summer Research Conference: Statistical Analysis of Measurement Error Models and Applications, Arcata, CA. 


\section{ACCEPTED MANUSCRIPT}

Sorbom, D. (1974). A general method for studying differences in factor means and factor structures between groups. British Journal of Mathematical and Statistical Psychology, 27(2), 229-39.

Steiger, J. H. (1979) 'Factor Indeterminacy in the 1930's and the 1970's Some Interesting Parallels.” Psychometrika 44(2), 157-167.

Thurstone, L.L. (1947). Multiple Factor Analysis. Chicago: The University of Chicago Press.

Vandenberg, R. J., \& Lance, C. E. (2000). A review and synthesis of the measurement invariance literature: Suggestions, practices, and recommendations for organizational research. Organizational Research Methods, 3(1), pp.

Wicherts, J. M., Dolan, C. V., \& Hessen, D. J. (2005). Stereotype threat and group differences in test performance: A question of measurement invariance. Journal of Personality and Social Psychology, 89(5), 696-716.

Wicherts, J. M., Dolan, C. V., Hessen, D. J., Oosterveld, P., van Baal, G. C. M., Boomsma, D. I., et al. (2004). Are intelligence tests measurement invariant over time? Investigating the nature of the Flynn effect. Intelligence, 32(5), 509-537.

Wilson, E. B. (1928). Review of "The abilities of man, their nature and measurement", by C. Spearman. Science, 67, 244-248.

Wu, A. D., Li, Z., \& Zumbo, B. D. (2007). Decoding the Meaning of Factorial Invariance and Updating the Practice of Multi-group Confirmatory Factor Analysis: A Demonstration With TIMSS Data. Practical Assessment, Research \& Evaluation, 12(3), 1-26. 


\section{ACCEPTED MANUSCRIPT}

${ }^{\mathrm{i}}$ The problem that we will be discussing is logically equivalent regardless of the dimensionality of factor representation. We will, therefore, restrict our attention throughout the manuscript to the unidimensional case, thereby effectively eliminating the weakest level of invariance, configural invariance, from consideration.

${ }^{i i}$ In the absence of additional constraints, certain parameters cannot be recovered. The details, however, are not central to the issues under consideration.

${ }^{\text {iii }}$ For a detailed history, see Maraun (2003).

${ }^{\text {iv }}$ We are well aware that there are those who will be wont to claim that, especially in the context of a technical discipline such as statistics, "mere language", notably, terminological choice, is of little consequence. We suggest that both the histories of the natural and mathematical sciences have shown this view to be naive. Mach (1960) allocates roughly forty pages to the task of demonstrating that Newton's concept of mass contains a circularity, explaining why such poorly engineered concepts cannot provide a reasonable basis for scientific investigation within physics, and offering an alternative definition that removes the circularity. One of Einstein's many contributions to physics was to point out the confusion that existed in regard the physicist's employment of the concept simultaneity. Essentially, he pointed out that the standard employment of the concept was unproblematic for events occurring at close proximity, but breaks down in the case of events occurring at a great distance from each other (Waismann, 1965).

${ }^{\mathrm{v}}$ It is not uncommon to encounter claims to the effect that $\mathbf{\Lambda}^{\prime} \boldsymbol{\Sigma}^{-1} \mathbf{X}$ is the common factor to $\mathbf{X}$, or, alternatively, that it is essentially the common factor to $\mathbf{X}$. But this is a fallacy. No random variable that satisfies $C_{u l f}$, hence, no random variable that is a common factor to $\mathbf{X}$, can be a linear transformation of $\mathbf{X}$. That is, there does not exist a vector $\mathbf{t}$ such that $\mathbf{t}^{\prime} \mathbf{X}$ satisfies $C_{u l f}$ (that a common factor is a latent variable means, simply, that it is not a function of $\mathbf{X}$ ). Proof Let $\boldsymbol{\delta}=\mathbf{X}-\boldsymbol{\tau}-\boldsymbol{\Lambda} \xi$. By model requirement (ulf3), $\mathrm{C}(\boldsymbol{\delta})=\boldsymbol{\Sigma}-\boldsymbol{\Lambda} \boldsymbol{\Lambda}^{\prime}=\boldsymbol{\Theta}_{\boldsymbol{\delta}}, \boldsymbol{\Theta}_{\boldsymbol{\delta}}$ diagonal and positive definite, hence, of rank $p$. Now, if, for some $\mathbf{t}, \xi=\mathbf{t}^{\prime} \mathbf{X}$, then $\boldsymbol{\Lambda}=\boldsymbol{\Sigma} \mathbf{t}$ and $\mathrm{C}(\boldsymbol{\delta})=\boldsymbol{\Sigma}-\boldsymbol{\Sigma} \mathbf{t} \mathbf{t}^{\prime} \boldsymbol{\Sigma}=\boldsymbol{\Sigma}-\boldsymbol{\Sigma} \mathbf{t}\left(\mathbf{t}^{\prime} \boldsymbol{\Sigma} \mathbf{t}\right)^{-1} \mathbf{t}^{\prime} \boldsymbol{\Sigma}=$ $\Sigma^{1 / 2}\left(\mathbf{I}-\Sigma^{1 / 2} \mathbf{t}\left(\mathbf{t}^{\prime} \boldsymbol{\Sigma} \mathbf{t}\right)^{-1} \mathbf{t}^{\prime} \Sigma^{1 / 2}\right) \Sigma^{1 / 2}$. But $\mathbf{I}-\boldsymbol{\Sigma}^{1 / 2} \mathbf{t}\left(\mathbf{t}^{\prime} \boldsymbol{\Sigma} \mathbf{t}\right)^{-1} \mathbf{t}^{\prime} \boldsymbol{\Sigma}^{1 / 2}$ is idempotent and of rank $(p-1)$. Hence, C( $\left.\boldsymbol{\delta}\right)$ is of rank $(p-1)$, which contradicts (ulf3) (McDonald, 1977).

${ }^{\text {vi }}$ By "external variable" we mean simply a variable that is not contained in $\mathbf{X}$. 